\title{
Noncommutative Standard Model in Top Quark Sector
}

\author{
Mojtaba Mohammadi Najafabadi 1 \\ School of Particles and Accelerators, \\ Institute for Research in Fundamental Sciences (IPM) \\ P.O. Box 19395-5531, Tehran, Iran
}

\begin{abstract}
In this article we aim to estimate the bounds on the noncommutative scale $\Lambda_{N C}$ and to extract the $95 \%$ exclusion contours for some $\theta_{\mu \nu}$ components using the recent measurements of the top quark width and the $W$ boson polarization in top pair events from CDF experiment at Tevatron.
\end{abstract}

\footnotetext{
${ }^{1}$ Email: mojtaba@mail.ipm.ir
} 


\section{Introduction}

The Standard Model (SM) of the particles has been found to be in good agreement with the present experimental data in many of its aspects. However, in the framework of the SM top quark is the only quark which has a mass in the same order as the electroweak symmetry breaking scale, $v \sim 246 \mathrm{GeV}$, whereas all other observed fermions have masses which are a tiny fraction of this scale. This huge mass might be a hint that top quark plays an essential role in the electroweak symmetry breaking. On the other hand, the reported experimental data from Tevatron on the top quark properties are still limited and no significant deviations from the Standard Model predictions has been seen. Several properties of the top quark have been already examined. They consist of studies of the $t \bar{t}$ production cross section, the top quark mass measurement, the measurement of $W$ helicity in the top decay, the search for FCNC and many other studies [1]. However, it is expected that top quark properties can be examined with high precision at the LHC due to very large statistics [2]. Since the dominant top quark decay mode is into a $W$ boson and a bottom quark, the $t W b$ coupling can be investigated accurately. Within the SM, the top quarks decay via electroweak interaction before hadronization. This important property is one of the consequences of its large mass. Hence, the spin information of the top quark is transferred to its decay daughters and can be used as a powerful mean for investigation of possible new physics.

There are many studies for testing the top quark decay properties at hadron colliders. For instance, the non-standard effects on the full top width have been investigated in the minimal supersymmetric standard model and in the technicolor model [3],[4],[5], [6], [7]. Some studies have been performed on the effects of anomalous $t W b$ couplings on the top width and some constraints have been applied on the anomalous couplings [8], 9], [10], [11],[12], [13].

The noncommutativity in space-time is a possible generalization of the usual quantum mechanics and quantum field theory to describe the physics at very short distances of the order of the Planck length, since the nature of the space-time changes at these distances 
(motivations to construct models on noncommutative space-time are coming from string theory, quantum gravity, Lorentz breaking [14],[15],[16],[17]). In the simplest case, the noncommutativity in space-time is described by a set of constant c-number parameters $\theta^{\mu \nu}$ or equivalently by an energy scale $\Lambda_{N C}$ and dimensionless parameters $C^{\mu \nu}$ :

$$
\left[\hat{x}_{\mu}, \hat{x}_{\nu}\right]=i \theta_{\mu \nu}=\frac{i}{\Lambda_{N C}^{2}} C_{\mu \nu}=\frac{i}{\Lambda_{N C}^{2}}\left(\begin{array}{cccc}
0 & -E_{1} & -E_{2} & -E_{3} \\
E_{1} & 0 & -B_{3} & B_{2} \\
E_{2} & B_{3} & 0 & -B_{1} \\
E_{3} & -B_{2} & B_{1} & 0
\end{array}\right)
$$

where $\theta_{\mu \nu}$ is a real antisymmetric tensor with the dimension of $[L]^{2}$. Here we have defined dimensionless electric and magnetic parameters $(\vec{E}, \vec{B})$ for convenience. We note that a space-time noncommutativity, $\theta_{0 i} \neq 0$, might lead to some problems with unitarity and causality [18], 19]. It has been shown that the unitarity can be satisfied for the case of $\theta_{0 i} \neq 0$ provided that $\theta^{\mu \nu} \theta_{\mu \nu}>0[20$. However for simplicity, in this article we take $\theta_{0 i}=0$ or equivalently $\vec{E}=0$.

A noncommutative version of an ordinary field theory can be obtained by replacing all ordinary products with Moyal $\star$ product defined as [21]:

$$
\begin{aligned}
(f \star g)(x) & =\left.\exp \left(\frac{i}{2} \theta^{\mu \nu} \partial_{\mu}^{y} \partial_{\nu}^{z}\right) f(y) g(z)\right|_{y=z=x} \\
& =f(x) g(x)+\frac{i}{2} \theta^{\mu \nu}\left(\partial_{\mu} f(x)\right)\left(\partial_{\nu} g(x)\right)+O\left(\theta^{2}\right) .
\end{aligned}
$$

The approach to the noncommutative field theory based on the Moyal product and Seiberg-Witten maps allows the generalization of the standard model to the case of noncommutative space-time, keeping the original gauge group and particle content [22],[23], [24],[25],26], [27]. Seiberg-Witten maps relate the noncommutative gauge fields and ordinary fields in commutative theory via a power series expansion in $\theta$. Indeed the noncommutative version of the Standard Model is a Lorentz violating theory, but the Seiberg Witten map shows that the zeroth order of the theory is the Lorentz invariant Standard Model. The effects of noncommutative space-time on some rare decay, collider processes, 
leptonic decay of the $W$ and $Z$ bosons and additional phenomenological results have been presented in [28], 229, 30], 31], 332, 33], 34], 35], 336, 337, [39] and some limits have been set on noncommutative scale.

The aim of this article is to estimate the bounds on the noncommutative scale $\Lambda_{N C}$ and to estimate the $95 \%$ exclusion contours for $\vec{B}$ using the current measurements of the top quark width and the $W$ boson polarization in the $t \bar{t}$ events from CDF experiment at Tevatron. In Section 2, a short introduction for the noncommutative standard model (NCSM) is given. Section 3 is dedicated to review the $W$ boson polarization in the top quark events. Section 4 presents the noncommutative effects on the top quark width and limit on $\Lambda_{N C}$ from current measured top width. Section 5 gives the limits on $\Lambda_{N C}$ and $\vec{B}$ using $W$ boson polarization. Finally, Section 6 concludes the paper.

\section{The noncommutative standard model (NCSM)}

The action of the NCSM can be obtained by replacing the ordinary products in the action of the classical SM by the Moyal products and then matter and gauge fields are replaced by the appropriate Seiberg-Witten expansions. The action of NCSM can be written as:

$$
S_{N C S M}=S_{\text {fermions }}+S_{\text {gauge }}+S_{\text {Higgs }}+S_{\text {Yukawa }}
$$

This action has the same structure group $S U(3)_{C} \times S U(2)_{L} \times U(1)_{Y}$ and the same fields number of coupling parameters as the ordinary SM. The approach which has been used in [23], 24],[25], [26] to build the NCSM is the only known approach that allows to build models of electroweak sector directly based on the structure group $S U(2)_{L} \times U(1)_{Y}$ in a noncommutative background. The NCSM is an effective, anomaly free, noncommutative field theory [40],[41].

We just consider the fermions (quarks and leptons). The fermionic matter part in a very compact way is:

$$
S_{\text {fermions }}=\int d^{4} x \sum_{i=1}^{3}\left(\overline{\hat{\Psi}}_{L}^{(i)} \star\left(i \not{D} \widehat{\Psi}_{L}^{(i)}\right)\right)+\int d^{4} x \sum_{i=1}^{3}\left(\overline{\widehat{\Psi}}_{R}^{(i)} \star\left(i \not \widehat{D} \widehat{\Psi}_{R}^{(i)}\right)\right),
$$


where $i$ is generation index and $\Psi_{L, R}^{i}$ are:

$$
\Psi_{L}^{(i)}=\left(\begin{array}{c}
L_{L}^{i} \\
Q_{L}^{i}
\end{array}\right), \Psi_{R}^{(i)}=\left(\begin{array}{c}
e_{R}^{i} \\
u_{R}^{i} \\
d_{R}^{i}
\end{array}\right)
$$

where $L_{L}^{i}$ and $Q_{L}^{i}$ are the well-known lepton and quark doublets, respectively. The SeibergWitten maps for the noncommutative fermion and vector fields yield:

$$
\begin{aligned}
\widehat{\psi} & =\widehat{\psi}[V]=\psi-\frac{1}{2} \theta^{\mu \nu} V_{\mu} \partial_{\nu} \psi+\frac{i}{8} \theta^{\mu \nu}\left[V_{\mu}, V_{\nu}\right] \psi+O\left(\theta^{2}\right) \\
\widehat{V_{\alpha}} & =\widehat{V_{\alpha}}[V]=V_{\alpha}+\frac{1}{4} \theta^{\mu \nu}\left\{\partial_{\mu} V_{\alpha}+F_{\mu \alpha}, V_{\nu}\right\}+O\left(\theta^{2}\right),
\end{aligned}
$$

where $\psi$ and $V_{\mu}$ are ordinary fermion and gauge fields, respectively. Noncommutative fields are denoted by a hat. For a full description and review of the NCSM, see [23], [24], [25], [26]. The $t\left(p_{1}\right) \rightarrow W(q)+b\left(p_{2}\right)$ vertex in the NCSM up to the order of $\theta^{2}$ can be written as [32, [36]:

$$
\begin{aligned}
\Gamma_{\mu, N C} & =\frac{g V_{t b}}{\sqrt{2}}\left[\gamma_{\mu}+\frac{1}{2}\left(\theta_{\mu \nu} \gamma_{\alpha}+\theta_{\alpha \mu} \gamma_{\nu}+\theta_{\nu \alpha} \gamma_{\mu}\right) q^{\nu} p_{1}^{\alpha}\right. \\
& \left.-\frac{i}{8}\left(\theta_{\mu \nu} \gamma_{\alpha}+\theta_{\alpha \mu} \gamma_{\nu}+\theta_{\nu \alpha} \gamma_{\mu}\right)\left(q \theta p_{1}\right) q^{\alpha} p_{1}^{\nu}\right] P_{L}
\end{aligned}
$$

where $P_{L}=\frac{1-\gamma_{5}}{2}$ and $q \theta p_{1} \equiv q^{\mu} \theta_{\mu \nu} p_{1}^{\nu}$. This vertex is similar to the vertex of $W$ decays into a lepton and anti-neutrino [37]. However, one should note that due to the ambiguities in the SW maps there are additional terms in the above vertex. Since they will not affect the results, we have ignored them 38 .

\section{$3 \quad W$ boson polarization in top events}

This section presents the observables used to measure the polarization of the $W$ boson. The real $W$ in the $t \rightarrow W+b$ decay can be produced with a longitudinal, left-handed or right-handed helicity. The corresponding probabilities are $F_{0}, F_{L}$ and $F_{R}$, respectively, whose SM expectations at tree level in the zero b-mass approximation are: 


$$
\begin{aligned}
F_{0} & =\frac{\Gamma\left(t \rightarrow W_{0} b\right)}{\Gamma(t \rightarrow W b)}=\frac{m_{t}^{2}}{m_{t}^{2}+2 m_{W}^{2}}=0.703 \\
F_{L} & =\frac{\Gamma\left(t \rightarrow W_{L} b\right)}{\Gamma(t \rightarrow W b)}=\frac{2 m_{W}^{2}}{m_{t}^{2}+2 m_{W}^{2}}=0.297 \\
F_{R} & =\frac{\Gamma\left(t \rightarrow W_{R} b\right)}{\Gamma(t \rightarrow W b)}=0.000
\end{aligned}
$$

where $m_{t}$ and $m_{W}$ are the top and $W$ masses in GeV. $\Gamma(t \rightarrow W b)$ is the top quark width. We have the restriction $F_{0}+F_{L}+F_{R}=1$. Since massless particles must be lefthanded in the SM, right-handed $W$ bosons do not exist in the zero b-mass approximation, due to angular momentum conservation. Including QCD and electroweak radiative corrections, finite width corrections and non-zero b-quark mass induces small variations: $F_{0}=0.695, F_{L}=0.304$ and $F_{R}=0.001$ for $m_{t}=175 \mathrm{GeV} / \mathrm{c}^{2}$ [42]. Because the top quark is very heavy, $F_{0}$ is large and the top decay is the only significant source of longitudinal $\mathrm{W}$ bosons. Deviations of $F_{0}$ from its SM value would bring into question the validity of the Higgs mechanism of the spontaneous symmetry breaking, responsible for the longitudinal degree of freedom of the massive gauge bosons. Any deviation of $F_{R}$ from zero could point to a non-Standard Model couplings such as $W t b$ anomalous couplings or new couplings coming from space-time noncommutativity introduced in the last section. The best way to access particle spin information is to measure the angular distribution of its decay products, thereby called spin analyzers. As an example, the charged lepton from the decay of longitudinally polarized $W$ boson tends to be emitted transversally to the $W$ boson direction, due to angular momentum conservation. Similarly, the charged lepton from a left-handed (right-handed) $W$ boson is preferentially emitted in the opposite (same) $W$ boson direction. By definition of $\theta_{l}^{*}$ to be the angle between the charged lepton direction in the $W$ boson rest frame and the $W$ direction in the top quark rest frame, the normalized differential decay rate can be expressed as the following [43]:

$$
\frac{1}{\Gamma} \frac{d \Gamma}{d \cos \theta_{l}^{*}}=\frac{3}{8}\left(1+\cos \theta_{l}^{*}\right)^{2} F_{R}+\frac{3}{8}\left(1-\cos \theta_{l}^{*}\right)^{2} F_{L}+\frac{3}{4}\left(\sin \theta_{l}^{*}\right)^{2} F_{0} .
$$

The measured values of the fractions $F_{0}$ and $F_{R}$ of longitudinally polarized and righthanded $W$ bosons in top quark decays using data collected with the CDF II detector(the 
data set used in the analysis corresponds to an integrated luminosity of approximately $955 \mathrm{pb}^{-1}$ ) are as the following [44]:

$$
\begin{aligned}
& F_{0}=0.59 \pm 0.12(\text { stat })_{-0.06}^{+0.07}(\text { syst }) \\
& F_{R}=-0.03 \pm 0.06(\text { stat })_{-0.03}^{+0.04}(\text { syst }) \\
& F_{R} \leq 0.1 \text { at } 95 \% \text { Confidence Level }
\end{aligned}
$$

\section{The noncommutative effects on the top quark width}

Using the introduced Feynman rule for the $W t b$ vertex in the NCSM in eq.7, the decay rate is easily evaluated. The decay rate in the top quark rest frame, which contains the noncommutative effects can be expressed as the following [36]:

$$
\Gamma(t \rightarrow W b)=\frac{\left|V_{t b}\right|^{2}}{16 \pi m_{t}}\left(\frac{g}{2 \sqrt{2} m_{W}}\right)^{2} \lambda^{1 / 2}\left(1, \frac{m_{W}^{2}}{m_{t}^{2}}, \frac{m_{b}^{2}}{m_{t}^{2}}\right)\left[A_{S M}+A_{N C}\right]
$$

where,

$$
\begin{aligned}
& A_{S M}=2\left[m_{W}^{2}\left(m_{t}^{2}+m_{b}^{2}-m_{W}^{2}\right)+\left(\left(m_{t}^{2}-m_{b}^{2}\right)^{2}-m_{W}^{4}\right)\right] \\
& A_{N C}=\frac{m_{W}^{2}}{12 \Lambda_{N C}^{4}}|\vec{B}|^{2}\left(5 m_{b}^{2}+m_{t}^{2}-m_{W}^{2}\right)\left[m_{b}^{4}+\left(m_{t}^{2}-m_{W}^{2}\right)^{2}-2 m_{b}^{2}\left(m_{t}^{2}+m_{W}^{2}\right)\right]
\end{aligned}
$$

One should note that in the above relation we have set $\theta_{i 0}=0$ and the terms with $\theta_{i j}$ or equivalently $\vec{B}$ are kept.

The SM prediction for the top quark lifetime is around $4 \times 10^{-25} \mathrm{~s}$ which corresponds to the top quark width of $1.5 \mathrm{GeV}$. It is notable that because of the limited resolutions of the experiments, it is very difficult to measure this very short lifetime or the corresponding width. However, we are able to set an upper limit on the top quark width from the available data from Tevatron. In [45] an upper limit has been set on the top quark width using a likelihood fit to the reconstructed top mass distribution. In the analysis the lepton+jets channel of $t \bar{t}$ candidates, in which one of two W-bosons decays to $l \nu_{l}$ while the other decays to $q \bar{q}$, is used to reconstruct the top quark mass. Finally, the estimated upper bound on the top quark width is $12.7 \mathrm{GeV}$ with $95 \%$ C.L. This is corresponding to the lower limit of $5.2 \times 10^{-26} \mathrm{~s}$ for the top quark lifetime. 
The measured upper limit on the top quark width and eq11 lead to the following bound on $\Lambda_{N C}$ :

$$
\Lambda_{N C} \geq 624 \mathrm{GeV} \text { for }|\vec{B}|^{2}=1 \text { with } 95 \% \text { C.L. }
$$

\section{The effects of noncommutativity on $W$ boson po- larization}

The noncommutative corrections to $F_{0}$ and $F_{R}$ can be calculated using the general $W t b$ vertex given by eq[7. The noncommutative corrections to $F_{0}$ are proportional to $\left|\theta_{i 0}\right|^{2}$ and there is no contribution from $\left|\theta_{i j}\right|$. Therefore $F_{0}$ can not provide us any information about $\left|\theta_{i j}\right|$.

The noncommutative corrections to $F_{R}$ can be evaluated by calculation the corrections to $\Gamma\left(t \rightarrow W_{R} b\right)$ :

$$
\begin{aligned}
\Gamma_{N C}\left(t \rightarrow W_{R} b\right) & =\frac{\left|V_{t b}\right|^{2}}{16 \pi m_{t}}\left(\frac{g}{2 \sqrt{2}}\right)^{2} \lambda^{1 / 2}\left(1, \frac{m_{W}^{2}}{m_{t}^{2}}, \frac{m_{b}^{2}}{m_{t}^{2}}\right) \Delta_{R} \\
\Delta_{R} & =\frac{m_{t}^{4}}{48}\left(m_{t}^{2}+m_{b}^{2}-m_{W}^{2}\right) \times \sum_{i=1}^{2} \theta^{i \mu} \theta_{\mu}^{i}
\end{aligned}
$$

and $F_{R}$ is:

$$
F_{R}=\frac{\Gamma_{N C}\left(t \rightarrow W_{R} b\right)}{\Gamma(t \rightarrow W b)}
$$

In obtaining the above relation we have neglected the contributions from $\theta_{i 0}$. The sum over $i$ is from one to two which is due the fact that the top quark is only restricted to decay into right-handed W-boson. Obviously, even in the limit of vanishing b-quark mass and neglecting the QCD and electroweak corrections, there are non-zero contributions to the $F_{R}$ from noncommutativity. $F_{R}$ is very sensitive to $\Lambda_{N C}$ for low values. The maximum value of $F_{R}$ which is equal to one corresponds to $\Lambda_{N C} \sim 870 \mathrm{GeV}$. With the increase in $\Lambda_{N C}, F_{R}$ approaches the SM value which is zero. The combination of the upper limit on $F_{R}$ measured by CDF, mentioned in Section 3, and the above relation leads to:

$$
\Lambda_{N C} \geq 1550 \mathrm{GeV} \text { for }|\vec{B}|^{2}=1 \text { with } 95 \% \text { C.L. }
$$




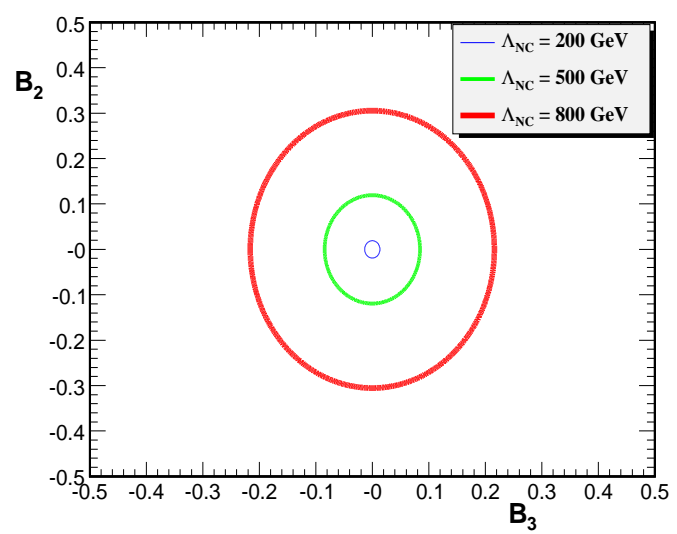

Figure 1: The 95\% C.L. exclusion contours on $B_{2}, B_{3}$ for different values of $\Lambda_{N C}$.

This bound is higher than the one obtained in [28] (from $Z \gamma$ production at the Tevatron and the LHC) and the bound obtained in [34] from SM forbidden decays which is $\Lambda_{N C}>1$ $\mathrm{TeV}$. It is noticeable that any better measurement on the upper bound of $F_{R}$ causes to a higher limit on $\Lambda_{N C}$.

The noncommutative corrections to $F_{R}$ and the CDF upper also limit provides the $95 \%$ C.L. exclusion contours on $B_{1}, B_{2}, B_{3}$ for different values of $\Lambda_{N C}(200,500,800 \mathrm{GeV})$. These contours are presented in Figure 1, According to the form of variation of $\vec{B}$ with changing the frame (such as the Lorentz transformation of magnetic field in electrodynamics), the change of frame leads to slightly lower limits.

The estimated bounds are comparable with the bounds estimated in [28] and lead to: $\left|\theta_{i j}\right| \leq 10^{-7} \mathrm{GeV}^{-2}$.

\section{Conclusion}

The recent measurements of the top quark width and $W$ boson polarization have been used to estimate the noncommutative scale $\Lambda_{N C}$ and to estimate the $95 \%$ exclusion contours on $B_{1,2,3}$. The extracted limits confirms the limits obtained by other studies . The $95 \%$ bound on $\Lambda_{N C}$ from the measured top quark width and $W$ polarization are 
$\Lambda_{N C} \geq 625 \mathrm{GeV}, \Lambda_{N C} \geq 1550 \mathrm{GeV}$ (assuming $|\vec{B}|^{2}=1$ ), respectively. The obtained limit on $\Lambda_{N C}$ from $W$ boson polarization is higher than the one obtained from the top width and also is higher than the limits obtained in [28] from $Z_{\gamma}$ production and the limits estimated in 34 from SM forbidden decays. The $95 \%$ exclusion contours on $B_{1,2,3}$ lead to $\left|\theta_{i j}\right| \leq 10^{-7} \mathrm{GeV}^{-2}$.

\section{Acknowledgments}

The author would like to thank Prof. H. Arfaei for fruitful discussions.

\section{References}

[1] A. Arbuzov et al., arXiv:0705.3251].

[2] M. Beneke et al., hep-ph/0003033.

[3] J. j. Cao, R. J. Oakes, F. Wang and J. M. Yang, Phys. Rev. D 68, 054019 (2003).

[4] J. Yang, C. Li, Phys. Lett. B 320, 117 (1994).

[5] A. Brandenburg, M. Maniatis, Phys. Lett. B 545, 139 (2002).

[6] X. Wang, Q. Zhang, Q. Qiao, Phys. Rev. D 71, 014035 (2005).

[7] M. Mohammadi Najafabadi and S. Paktinat Mehdiabadi, arXiv:0803.1287.

[8] E. Boos, L. Dudko, T. Ohl, Eur. Phys. J. C 11, 473 (1999).

[9] J. A. Aguilar-Saavedra, J. Carvalho, N. Castro, A. Onofre, F. Veloso, Eur. Phys. J. C 50, 519 (2007).

[10] M. Mohammadi Najafabadi, J. Phys. G 34, 39 (2007).

[11] B. Grzadkowski and M. Misiak, arXiv:0802.1413]. 
[12] M. Mohammadi Najafabadi, JHEP 03, 24 (2008).

[13] J. A. Aguilar-Saavedra, arXiv:0803.3810.

[14] M. R. Douglas and N. A. Nekrasov, Rev. Mod. Phys. 73, 977 (2002).

[15] A. Connes, M. R. Douglas, and A. Schwarz, JHEP 9802, 003 (1998).

[16] F. Ardalan, H. Arfaei and M. M. Sheikh-Jabbari, hep-th/9803067.

[17] F. Ardalan, H. Arfaei and M. M. Sheikh-Jabbari, JHEP 9902, 016 (1999).

[18] J. Gomis, T. Mehen, Nucl. Phys. B 591, 265 (2000).

[19] M. Chaichian, A.Demichev, P. Presnajder, Eur. Phys. J. C 20, 767 (2001).

[20] S.M. Carrol, J.A. Harvey, V.A. Kostelecky, C.D. Lane and T. Okamoto, Phys. Rev. Lett. 87, 141601 (2001).

[21] M.R. Douglas, N.A. Nekrasov, Rev. Mod. Phys. 73, 977 (2001).

[22] N. Seiberg, E. Witten , JHEP 09, 032 (1999).

[23] J. Madore, S. Schraml, P. Schupp, J. Wess, Eur. Phys. J. C 16, 161 (2000).

[24] B. Jurco, S. Schraml, P. Schupp, J. Wess, Eur. Phys. J. C 17, 521 (2000).

[25] B. Jurco, P. Schupp, J. Wess, Nucl. Phys. B 604, 148 (2001).

[26] X. Calmet, B. Jurco, P. Schupp, J. Wess, M. Wohlgenannt, Eur. Phys. J. C 23, 363 (2002).

[27] T. Ohl and J. Reuter, Phys. Rev. D 70, 076007 (2004).

[28] A. Alboteanu, T. Ohl, R. Ruckl, Phys. Rev. D 74, 096004 (2006).

[29] M. Haghighat, M. M. Ettefaghi, M. Zeinali, Phys. Rev. D 73, 013007 (2005).

[30] P. Schupp, J. Trampetic, J, Wess and G. Raffelt, Eur. Phys. J. C 36, 405 (2004). 
[31] C.P. Martin and C. Tamarit, JHEP 02, 066 (2006).

[32] M. Mohammadi Najafabadi, Phys. Rev. D 74, 025021 (2006).

[33] J. Trampetic, arXiv:0802.2030].

[34] M. Buric, D. Latas, V. Radovanovic and J. Trampetic, Phys. Rev. D 77, 045031 (2008).

[35] B. Melic, K. W. Behr, N.G. Deshpande, G. Duplancic, P. Schupp, J. Trampetic and J. Wess, Eur. Phys. J. C 29, 441 (2003).

[36] N. Mahajan, Phys. Rev. D 68, 095001 (2003).

[37] E.O. Iltan, Phys. Rev. D 66, 034011 (2002).

[38] A. Alboteanu, T. Ohl and R. Ruckl, Phys. Rev. D 76, 105018 (2007).

[39] H. Arfaei, M. H. Yavartanoo, hep-th/0010244.

[40] C.P. Martin, Nucl. Phys. B 652, 72 (2003).

[41] F. Brandt, C.P. Martin and F. Ruiz, JHEP 07, 068 (2003).

[42] H. S. Do, S. Groote, J. G. Korner and M. C. Mauser, Phys. Rev. D 67, 091501 (2003).

[43] G.L. Kane, G.A. Ladinsky and C.-P. Yuan, Phys. Rev. D 45, 124 (1992).

[44] T. Chwalek, arXiv:0705.2966].

[45] CDF collaboration, A Limit on the Top Quark Width and the Lifetime using the Template Method in the Lepton plus Jets Channel at CDF II, CDF note 8953, August 10, 2007. 